\title{
Association of sleep duration and sleep quality with hypertension in oil workers in Xinjiang
}

\author{
Fen Yang ${ }^{1}$, Yuanyue Zhang ${ }^{1}$, Ruiying Qiu ${ }^{1}$, Ning Tao ${ }^{\text {Corresp. } 1,2}$ \\ ${ }^{1}$ School of public health, Xinjiang Medical University, Xinjiang, China \\ ${ }^{2}$ Clinical Postdoctoral Mobile Station, Xinjiang Medical University, Xinjiang, China \\ Corresponding Author: Ning Tao \\ Email address: 38518412@qq.com
}

Objective. The aim of this study is to explore sleep status and hypertension among oil workers in Xinjiang, China. It may provide new ideas and basis for the precise prevention and treatment of hypertension in occupational population. Methods. Sleep status and hypertension were investigated in 3040 workers by a multi-stage cluster sampling method in six oil field bases in Karamay City, Xinjiang. The Pittsburgh Sleep Quality Index was used to evaluate the sleep status of workers. Logistic regression was used to analyze the relationship between sleep duration and sleep quality, and hypertension. Stratified analysis was also performed. Results . Our results show: 1. Insufficient sleep duration $(O R=1.51,95 \% \mathrm{Cl}: 1.19-1.90)$ and poor sleep quality $(O R=1.78,95 \% \mathrm{Cl}: 1.33-2.38)$ were positively associated with hypertension . 2 . Stratified analysis indicated insufficient sleep duration was associated with increased risk of hypertension in females $(O R=1.54,95 \% \mathrm{Cl}$ : 1.16-2.04) than males $(O R=1.49,95 \% \mathrm{Cl}: 1.00-2.23)$, and the risk of hypertension in the group $\& \lambda \tau ; 30$ years old $(O R=9.03,95 \% \mathrm{Cl}: 2.32-35.15)$ was higher than that in the group of 30-45 years old $(O R=1.59,95 \% \mathrm{Cl}: 1.14-2.20)$. However, in the group $>45$ years old, sleeping $\& \gamma \tau$; $8 \mathrm{~h}$ was associated with increased risk of hypertension $(O R=3.36$, $95 \% \mathrm{Cl}: 1.42-7.91)$. Oil workers doing shift work had a higher risk of hypertension $(O R=1.55$, $95 \% \mathrm{Cl}: 1.16-2.07)$ to no shift work $(O R=1.48,95 \% \mathrm{Cl}: 1.02-2.15)$. The risk of hypertension in the group with $\& \lambda \tau ; 10$ years of service $(O R=4.08,95 \% \mathrm{Cl}: 1.92-8.83)$ was higher than that in the group with length of service of $10-20$ years $(O R=2.79,95 \% \mathrm{Cl}: 1.59-4.86)$. Poor sleep quality was associated with risk for hypertension in females $(O R=1.78,95 \% \mathrm{Cl}$ : 1.26-2.49), those doing shift work $(O R=1.70,95 \% \mathrm{Cl}: 1.17-2.47)$, those with length of service of $\& \gamma \tau ; 20$ years $(O R=1.64,95 \% \mathrm{Cl}: 1.18-2.27)$. with the risk of hypertension in the group 30-45 years old higher than that in the group $\& \gamma \tau$; 45 years old $\left(O R_{30-45 \text { years old }}=1.71\right.$, 95\% Cl: 1.10-2.66; $O R_{\text {\&үт;4 years old }}=1.60,95 \% \mathrm{Cl}$ : 1.09-2.34. Conclusion. Insufficient sleep duration and poor sleep quality are the potential factors affecting hypertension in Xinjiang oil workers. 
G।

$\mathrm{Ye}$

\section{Association of sleep duration and sleep quality with 2 hypertension in oil workers in Xinjiang}

3



\section{Abstract}

Objective. The aim of this study is to explore sleep status and hypertension among oil workers in Xinjiang, China. It may provide new ideas and basis for the precise prevention and treatment of hypertension in occupational population.

Methods. Sleep status and hypertension were investigated in 3040 workers by a multi-stage cluster sampling method in six oil field bases in Karamay City, Xinjiang. The Pittsburgh Sleep Quality Index was used to evaluate the sleep status of workers. Logistic regression was used to analyze the relationship between sleep duration and sleep quality, and hypertension. Stratified analysis was also performed.

Results. Our results show: 1 . Insufficient sleep duration $(O R=1.51,95 \% C I$ : $1.19-1.90)$ and poor sleep quality $(O R=1.78,95 \% C I: 1.33-2.38)$ were positively associated with hypertension. 2. Stratified analysis indicated insufficient sleep duration was associated with increased risk of hypertension in females $(O R=1.54,95 \% C I: 1.16-2.04)$ than males $(O R=1.49,95 \% C I: 1.00-2.23)$, and the risk of hypertension in the group $<30$ years old $(O R=9.03,95 \% C I: 2.32-35.15)$ was higher than that in the group of 30-45 years old $(O R=1.59,95 \% C I: 1.14-2.20)$. However, in the group $>$ 45 years old, sleeping $>8 \mathrm{~h}$ was associated with increased risk of hypertension $(O R=3.36$, $95 \% C I: 1.42-7.91)$. Oil workers doing shift work had a higher risk of hypertension $(O R=1.55$, 95\%CI: 1.16-2.07) to no shift work ( $O R=1.48,95 \% C I: 1.02-2.15)$. The risk of hypertension in the group with $<10$ years of service $(O R=4.08,95 \% C I$ : 1.92-8.83) was higher than that in the group with length of service of $10-20$ years $(O R=2.79,95 \% C I: 1.59-4.86)$. Poor sleep quality was associated with risk for hypertension in females $(O R=1.78,95 \% C I: 1.26-2.49)$, those doing shift work ( $O R=1.70,95 \% C I$ : $1.17-2.47)$, those with length of service of $>20$ years $(O R=1.64,95 \% C I$ : 1.18-2.27). with the risk of hypertension in the group 30-45 years old higher than that in the group $>45$ years old $\left(O R_{30-45}\right.$ years old $=1.71,95 \% C I: 1.10-2.66 ; O R_{>45}$ years old $=1.60,95 \% C I: 1.09-2.34$. 
38 Conclusion. Insufficient sleep duration and poor sleep quality are the potential factors affecting 39 hypertension in Xinjiang oil workers.

40

41 Introduction

42 Hypertension is a non-communicable disease and the most important risk factor leading to death 43 from cardiovascular disease. At present, although the etiology of hypertension is unclear, factors 44 identified as influencing hypertension include age, gender, race, lack of exercise, obesity, sodium 45 intake, alcohol, and occupational stress (Bergmann et al., 2014). In recent years, some studies have 46 found that sleep is also closely related to the occurrence of hypertension. Sleep has important 47 effects on the cardiovascular system function (Wang et al., 2017), as well as on physiological pathology (Christina et al., 2018). However, the rapid development of human society and changes in lifestyle have led to a decrease in average sleep duration and sleep quality (Zheng et al., 2014). In line with the National Health Interview Survey, short sleep duration is associated with an increased risk of hypertension among American adults, and this relationship is dependent on age and BMI (Oluwatimilehin et al., 2019). Wu et al. (2016) conducted a recent survey on hypertension in daytime and nighttime. In that study, the results showed that only males' sleep duration was associated with risk of hypertension. On the other hand, studies by Wu et al. (2016) found that lack of sleep associated with hypertension is prevalent only in females. These contrary results suggest there is a complex and controversial relationship between sleep and hypertension. Oil workers are a specific occupational group often involving shift work. Sleep problems often occur when there is a serious imbalance between the natural circadian rhythm and the shift system. Previous studies have shown that not only is shift work associated with adverse health effects by disturbing circadian rhythm, but may also impact sleep quality and be linked with diabetes and hypertension (Choi et al., 2019). Wang et al. (2014) conducted statistical analysis of the health status of oil workers in Ningxia, China, finding the prevalence of hypertension of $21.43 \%$. Such a high incidence suggests improving the sleep status and controlling for the risk of hypertension among desert oil workers is a matter of urgency, owing to the specific characteristics of this occupational group. Oil fields are often located in remote areas, including in deserts and off shore. As such, they are often affected by challenging weather conditions. Moreover, oil rigs typically operate 24 hours a day, thereby requiring shift work with oil field workers often working one week on and one week off.

Therefore, this cross-sectional study set out to investigate the sleep status of Chinese oil workers with the aim of exploring the association between sleep duration and sleep quality on hypertension. Confounding factors were analyzed by a logistic regression model. Sleep duration and sleep quality, and their association with hypertension were further investigated by stratified analysis, which provided a more comprehensive theoretical basis for identifying preventive measures of hypertension among oil workers in Xinjiang.

Materials \& Methods

\section{Study setting and participants}


77 According to $N=\mu_{\alpha}^{2} \rho(1-\rho) / \delta^{2}$ [ $\rho$ is the prevalence of hypertension (according to the research 78 results of LU et al, the value is $37 \%$ ), $\alpha=0.05$ (two-sided), $\delta=0.018$ ] that $\mathrm{N}=2763$. Taking into 79 account the censorship of 10\%-20\%, 3100 oil workers were finally selected. Cluster sampling was 80 used to select the study population. A cross-sectional survey of 3,100 oil workers in six oil field 81 bases in Karamay, Xinjiang, China, was conducted. One district (Karamay District) was randomly 82 selected from four districts (Karamay District, Baijiantan District, Wuerhe District, and Dushanzi 83 District) under the jurisdiction of Karamay City. Sixteen oil fields in this area were numbered $8401 \sim 16$, according to the random number table method. Six oil fields corresponding to the first 85 column of row 27 in the random number table were selected. All participants provided written 86 informed consent to a questionnaire survey. Excluding 33 with incomplete questionnaires and 27 87 oilfield workers who failed to measure blood pressure by professional doctors in Karamay Central 88 Hospital, 3,040 participants were finally included. The inclusion criteria were oil field workers 89 aged between 20 and 60 with work experience of at least one year who agreed to participate in this 90 survey. The exclusion criteria were those with severe organic diseases (that occurs in a certain 91 organ or a certain tissue system of the body caused by a variety of reasons, which causes permanent 92 damage to the organ or tissue system), mental illness, and genetic diseases. This study was 93 approved by the Ethics Review Committee of the First Affiliated Hospital of Xinjiang Medical 94 University (Ethics number: 2015006).

\section{Study methods}

\section{Socio-demographic characteristics}

97

Each subject participated in a structured questionnaire survey, which included factors such as gender (female, male), age ( $<30,30-45,>45$ years old), ethnicity (Han, others), marital status (unmarried, married, divorced/widow/widower), income ( $<5000 \mathrm{RMB}, \geq 5000 \mathrm{RMB}$ ), educational level (below high school, college level or above), job status (junior level, intermediate level, senior level), length of service $(<10,10-20,>20$ years), smoking status (non-smoker, smoker: smoking $\geq 1$ cigarette per day for six months or more), alcohol consumption (non-drinker, drinker: drinking $\geq 2$ times a week with alcohol intake $\geq 50$ g per drinking session regularly $\geq 1$ year), BMI $(<18.5,18.5-24,>24)$, and shift work status (no shift work, shift work: regular working hours other than 10:00-19:00 and lasting for more than 1 year).

\section{Sleep status}

The Pittsburgh Sleep Quality Index (PSQI) was used to assess participants' subjective sleep quality and sleep duration (Buysse et al., 1989). The survey used the Chinese version of the Pittsburgh Sleep Quality Index (CPSQI), with a Cronbach's alpha of 0.89 and a KMO of 0.91 (Tsai et al., 2005). The CPSQI comprises seven factors and 19 items. Each item is scored from 0 to 3 according to a 4-level Likert scale. In this study, a CPSQI $\leq 5$ was considered good sleep quality, whereas a score of $>5$ was regarded as poor sleep quality. Sleep duration was classified into "insufficient" ( $<7 \mathrm{~h}$ ), "normal" (7-8 h), and "long" ( $>8 \mathrm{~h}$ ) based on previous reports (Tsai et al., 2005).

\section{Ascertainment of hypertension}

Blood pressure was measured by a professional doctor in the Physical Examination Department of Karamay Central Hospital. The blood pressure and diagnosis of hypertension in each participant 
117 were determined after measuring resting blood pressure three times during different times of the 118 same day. Individuals were defined as having hypertension if they met one of the following 119 standards: (1) systolic blood pressure $\geq 140 \mathrm{~mm} \mathrm{Hg}$ and/or diastolic blood pressure $\geq 90 \mathrm{~mm} \mathrm{Hg}$; 120 (2) self-reported hypertension diagnosed by a physician and current antihypertensive treatment 121 during the previous two weeks.

\section{Statistical analysis}

123 The raw data were organized in Excel sheets. All the statistical analyses of this research were 124 performed using SPSS version 24.0. The categorical data are tabulated with frequencies and 125 percentages, and a Pearson chi-square test was computed to compare differences between groups. 126 Logistic regressions were performed to evaluate the relationship between sleep duration and sleep 127 quality with hypertension. The level of significance was set at 0.05 .

\section{Results}

\section{Basic characteristics of the sample population}

130

The data of all 3,040 participants in this study are presented in Table 1 . The overall prevalence of hypertension was $15.33 \%$ among all participants. Participants reporting sleep of $<7 \mathrm{~h}$ accounted

132

133

134

135

136

137

138

139

140

141

142

143

144

145

146

147

148

149

150

151

152

153

154

155

156 for $26.51 \%$, of which $21.00 \%$ reported hypertension. Participants reporting poor sleep quality accounted for $78.28 \%$, of which $16.50 \%$ reported hypertension. Hypertension showed significant association with all characteristics except for income level in the chi-squared test $(P<0.001)$.

\section{Sleep duration, sleep quality, and hypertension}

After adjusting for confounding factors including gender, age, ethnicity, educational level, job status, marital status, income, smoking status, alcohol consumption, shift work status, and BMI, insufficient sleep duration and poor sleep quality showed a statistically significant association with hypertension $\left(O R_{\text {sleep duration }}=1.51,95 \% C I: 1.19-1.90 ; O R_{\text {sleep quality }}=1.78,95 \% C I: 1.33-2.38\right)$. The results indicated that age $\left(O R_{30-45}=2.00,95 \% C I: 1.03-3.91 ; O R>_{45}=2.91,95 \% C I\right.$ : 1.43-5.91), gender $(O R=2.08,95 \% C I: 1.53-2.81)$, shift work status $(O R=2.22,95 \% C I: 1.78-2.78)$, and length of service $\left(O R_{10-20}=2.34,95 \% C I: 1.43-3.81 ; O R>_{20}=3.87,95 \% C I: 2.40-6.24\right)$ were risk factors associated with hypertension $(P<0.001)$. Income $(O R=0.65,95 \% C I$ : $0.52-0.81)$ and ethnicity $(O R=0.61,95 \% C I: 0.50-0.79)$ were protective factors for hypertension $(P<0.01)$. The risk of hypertension was higher in women than in men $(P<0.001)$. The educational level, job status, marital status, smoking status, alcohol consumption, and BMI did not show significant impact associated with risk of hypertension $(P>0.05)$. According to Hosmer-Lemeshow goodness-of-fit test, $\chi^{2}=14.929, P=0.061>0.05$, suggesting that the model has a good degree of fit and calibration ability; The area under the curve of the model AUC is $0.782>0.75,95 \%$ CI: $0.758-0.801$, suggesting that the prediction model has a good distinguishing ability (Table 2).

\section{Stratified analysis}

The logistic regression analysis indicated that age, gender, shift work status, and length of service were risk factors for hypertension, so we stratified according to these factors and analyzed the effect of sleep duration and sleep quality on hypertension. Insufficient sleep duration was found to be associated with increased risk of hypertension in females $(O R=1.54,95 \% C I$ : 1.16-2.04) higher than males $(O R=1.49,95 \% C I$ : 1.00-2.23), with the risk of hypertension in the group $<30$ years 
157

158

159

160

161

162

163

164

165

166

167

168

169

170

171

172

173

174

175

176

177

178

179

180

181

182

183

184

185

186

187

188

189

190

191

192

193

194

195

196

old higher than that in the group 30-45 years old $\left(O R_{<30 \text { years old }}=9.03,95 \% C I: 2.32-35.15 ; O R\right.$ 3045 years old $=1.59,95 \% C I: 1.14-2.20)$. However, in the group $>45$ years old $(O R=3.36,95 \% C I: 1.42-$ 7.91), sleeping for $\square 8 \mathrm{~h}$ was associated with increased risk of hypertension, with the risk of hypertension in the group with $<10$ years of service being higher than that in the group with the length of service of $10-20$ years $\left(O R_{<10 \text { years }}=4.08,95 \% C I: 1.92-8.83 ; O R_{10-20 \text { years }}=2.29,95 \% C I\right.$ : 1.59-4.86). Oil workers doing shift work had risk of hypertension $(O R=1.55,95 \% C I$ : $1.16-2.07)$ to no shift work $(O R=1.48,95 \% C I$ : 1.02-2.15). Compared with a good sleep quality, poor sleep quality was shown to be associated with increased risk of hypertension in females $(O R=1.78$, $95 \% C I: 1.26-2.49)$, length of service $>20(O R=1.64,95 \% C I: 1.18-2.27)$, and shift work $(O R=1.70$, $95 \% C I: 1.17-2.47)$; with the risk of hypertension in the group 30-45 years old higher than that in the group $>45$ years old $\left(O R_{30-45 \text { years old }}=1.71,95 \% C I: 1.10-2.66 ; O R>45\right.$ years old $=1.60,95 \% C I$ : 1.09-2.34 (Table 3).

\section{Discussion}

In this cross-sectional study, the associations of sleep duration and sleep quality with the prevalence of hypertension among petroleum workers in Xinjiang were investigated. We adjusted for gender, age, length of service, shift status and other confounding factors, and performed logistic regression analysis to assess the relationship between sleep duration and sleep quality, and hypertension. We found that insufficient sleep duration and poor sleep quality are associated with hypertension in Xinjiang oil workers. Moreover, their associations also analyzed by stratification, our results showed that this phenomenon was found in different gender, age, length of service and shift status.

\section{Relationship between hypertension sleep and hypertension in oil workers}

Hypertension currently affects $26.4 \%$ of adults worldwide, and it is a leading risk factor for mortality (Huang et al., 2012). Published in the Lancet in 2017, Lu et al. (2017) conducted a cardiovascular risk screening in 31 provinces across China, with a cumulative screening of more than 1.7 million urban and rural residents aged 35 to 75 years old. The results showed that the detection rate of age-adjusted hypertension was $37 \%$. However, the prevalence of hypertension in Xinjiang oil workers was $15.3 \%$ in this study. This difference could be attributed to the following reasons: First of all, the prevalence of hypertension varies with the age composition of the population, increasing with a greater proportion of elderly people. In this study, the number of oil workers $<45$ years old in this survey was relatively large, accounting for about $67 \%$ of the sample population. Secondly, Karamay City had adopted a comprehensive "four-party linkage" prevention and control model integrated with hospitals, community health service agencies, disease prevention and control agencies, and residents in the management of chronic diseases. Consequently, remarkable results have been achieved, and it has already been recognized as the Chinese Model Region for Chronic Disease Management in 2019. In addition, the sleep status of specific occupational groups may result in different prevalence of hypertension depending on their working conditions and working environment (Magnavita et al., 2019). Nevertheless, the prevention and control of hypertension of oil workers still requires intervention. 
197 Most oil-producing fields in Xinjiang are located in remote areas of the Gobi Desert, with harsh

198

199

200

201

202

203

204

205

206

207

208

209

210

211

212

213

214

215

216

217

218

219

220

221

222

223

224

225

226

227

228

229

230

231

232

233

234

235

236

natural conditions. Oil field workers are not only affected by severe weather, but also are under pressure from long working hours together with an irregular shift system, resulting in lack of sleep (Tao et al., 2015). From a physiological basis under such conditions, dehydration and subsequent cardiovascular stress are more likely to occur (Zhang et al., 2019). While some studies have shown unclear association between sleep and hypertension, other studies have shown that sleep deprivation increases the sympathetic activity of the nervous system and changes the hypothalamus-pituitary-adrenal axis, resulting in increased cortisol levels, and elevation of blood pressure and heart rate, identifying poor sleep quality an important risk factor for hypertension (Bruno et al., 2013; Feng et al., 2019).

\section{Gender differences in the effect of sleep on hypertension}

There is, however, a growing recognition of gender disparity in sleep-wake and circadian rhythm disorders (Mong et al., 2016; Nishichi et al., 2013). Analyzing gender by stratification in our study confirms an increased prevalence of poor sleep quality and insufficient sleep duration in females, compare to males. An explanation for the gender difference could be that men and women have different occupational trajectories and different social support in the workplace (Hayes et al., 2014). Physiologically, hormonal changes in the female menstrual cycle, menopause, pregnancy, and postpartum affect the body's circadian rhythm and sleep architecture, leading to frequent sleep disturbances and worsening sleep quality. In addition, these hormonal changes are associated with depression, anxiety and irritability, which may additionally cause a deterioration in the quality of sleeping in females. In terms of psychosocial factors, work stress and family stress aggravate the development of sleep problems, including insomnia, and females are more likely to be vulnerable to effects caused by a stressful life, because of a greater share of household duties and taking care of children in addition to work, resulting in more sleep problems.

\section{Age differences in the effect of sleep on hypertension}

Further, sleep disturbance is common during the menopausal transition and its effect of sleep duration on hypertension is U-shaped (Kecklund et al., 2016). We revealed similar findings in our study after stratifying for age. We found insufficient sleep duration is linked to hypertension in those $<45$ years of age, while too long sleep duration leads to a similar result in those $>45$ years, consistent with the results of Kecklund et al (2016). A large number of studies have shown that insufficient sleep increases the excitability of the sympathetic nervous system and the reninangiotensin system. This may be related to increased catecholamine synthesis in the central nervous system, which young people are more sensitive to. On the other hand, the sleep duration of the elderly is affected by age-related sleep structure changes, compensatory daytime sleep, and drug side effects, resulting in an increase in the prevalence of hypertension (Lu et al., 2015).

\section{Shift work differences in the effect of sleep on hypertension}

In addition, it is well-recognized that long-term shifts of may have some adverse health outcomes (Manohar et al., 2017). Therefore, we analyzed whether there is a difference in the effect of shift work on the relationship between sleep and hypertension. We observed slightly increased association between sleep disturbances and 
237

238

239

240

241

242

243

244

245

246

247

248

249

250

251

252

253

254

255

256

257

258

259

260

261

262

263

264

265

266

267

268

269

270

271

272

\section{Conclusions}

274

275

276

extension of shift work. Studies have confirmed that shift work is associated with disruption of sleep patterns and circadian rhythms. In particular, night shifts can disturb chronobiological rhythms and reduce the secretion of melatonin, thus directly reducing the quality of sleep (Guo et al., 2013). Shift work also interferes with the quality of sleep, which can become chronic and remain even after exposure has ceased. It has been reported that even after retirement older workers who worked shifts have a worse sleep pattern than other retirees (Härmä et al., 2018). In these former workers, polysomnographic studies have demonstrated the existence of a direct relationship between the duration of shift work and the frequency of altered sleep patterns (Heath et al., 2016).

\section{Length of service differences in the effect of sleep on hypertension}

Finally, after stratification according to the length of service, we found decrease in length of service increases the risk of hypertension when sleep duration is insufficient. But poor sleep quality is only associated with oil workers who have worked $>10$ years. This is likely because the length of service is highly related to age, and most of those with shorter length of service are young and middle-aged (Elogue et al., 2014).

Based on the above findings, to prevent occurrence of hypertension, we recommend strengthening health education for oil workers, including the provision of guidelines to help them achieve an appropriate work-life balance to maintain their health and adopt good sleeping habits such as changing sleep patterns. At the same time, employers should improve the working environment, and establish an appropriate system to improve the quality of professional life for workers, taking into consideration that shift work has an adverse impact on health. Finally, considering hypertension patients, health promotion and education should be strengthened to help them achieve the best therapeutic effect, thereby improving their long-term quality of life and health.

Although this study has some important findings, several limitations should be acknowledged. First, the participants were oil workers working in desert areas, who may be prone to occupational stress due to their specific occupational particularities. Magnavita et al., 2017; and Garbarino et al., 2015 have suggested occupational stress is an important factor associated with sleep disorders. On the other hand, some studies have indicated that sleep is an important moderator of the relationship between stress and hypertension (Garbarino et al., 2019). Therefore, owing to the complex relationship between sleep and hypertension, we did not include the impact of occupational stress in this initial study. In a future study, we plan to explore the relationship among sleep, occupational stress, and hypertension through intermediary effect analysis. Second, sleep quality and duration are self-reported data. There were no standard cut-off values to judge normal or abnormal sleep duration, or good or poor sleep quality. Third, causality and temporality could not be ascertained because this study was a cross-sectional survey. And finally, because of the sample size, it cannot represent all the oil workers in China. Thus, further investigation of oil workers is warranted for further understanding of the relationship between sleep and hypertension.

our findings suggest that sleep disturbances are associated with the prevalence of hypertension among oil workers. Our study confirms that insufficient sleep duration and poor sleep quality are factors associated with hypertension. After stratification by gender, age, shift work status, and

Peer) reviewing PDF | (2020:06:50421:2:0:NEW 25 Mar 2021) 
277 length of service, when both insufficient sleep duration and poor sleep quality coexist, the 278 following were found: There is an increased prevalence of hypertension in females; the risk of 279 hypertension decreases with age and length of service; And the prevalence of hypertension in shift 280 workers is high. This study has identified a number of factors associated with hypertension in oil 281 workers doing shift work in relation to sleep and hypertension, providing valuable data that can be 282 used to draw up measures and guidelines to prevent and manage hypertension among this and other 283 occupational groups, especially those with shift work, and hypertension patients in general.

284

285

286

287

288

289

290

291

292

293

294

295

296

297

298

299

300

301

302

303

304

305

306

307

308

309

310

311

312

313

314

315

316

\section{Acknowledgements}

The authors thank all participants and investigators.

\section{References}

Bergmann N, Gyntelberg F, Faber J. 2014. The appraisal of chronic stress and the development of the metabolic syndrome: a systematic review of prospective cohort studies. Endocrine Connections 3(2): R55-80 DOI 10.1530/EC-14-0031.

Wang DM, Zhou Y, Guo YJ, Zhang RB, Li WZ, He MA, Zhang XM, Guo H, Yuan J, Wu TC, Chen WH. 2017. The effect of sleep duration and sleep quality on hypertension in middle-aged and older Chinese: The Dongfeng-Tongji cohort study. Sleep Medicine 40: 7883 DOI 10.1016/j. Sleep. 2017. 09. 024.

Christina BJ, Fernandez-Mendoza J. 2018. Insomnia, short sleep duration, and high blood pressure: Recent evidence and future directions for the prevention and management of hypertension. Current Hypertension Reports 20(6): 52 DOI 10.1007/s11906-018-0850-6.

Zheng LW, Chen Y, Chen F, Zhang P. 2014. Effect of acupressure on sleep quality of middleaged and elderly patients with hypertension. International Journal of Nursing Sciences 1(4): 334-338 DOI 10.1016/j. Ins. 2014. 10. 012.

Oluwatimilehin O, Okunowo HT, Helen T, Njesada NS, Solomon A. 2019. Age and body weight dependent association between sleep duration and hypertension in us adults: Findings from the 2014-2017 national health interview survey. Sleep health 5(5): 509-531 DOI 10.1016/j.sleh.2019.05.003.

Wu L, He Y, Jiang B, Liu M, Wang JH, Zhang D, Wang YY, Zeng J, Yao Y. 2016. Association between sleep duration and the prevalence of hypertension in an elderly rural population of china. Sleep Medicine 27-28: 92-98 DOI 10.1016/j.sleep.2016.08.015.

Wu WW, Wang WR, Guo YH, Xie YF, Liu XX, Chen XY, Zhang YT, Tan XD. 2019. Sleep quality, sleep duration, and their association with hypertension prevalence among lowincome oldest-old in a rural area of china: A population-based study. Journal of psychosomatic research 127: 109848 DOI 10.1016/j.jpsychores.2019.109848.

Choi WS, Lee J, Lee JY, Kim KY, Myong JP, Lee W. 2019. The effect of special medical examination for night shift workers and follow-up management against hypertension. International journal of environmental research and public health 16: 719 DOI 10.3390/ijerph16050719.

Wang Y, Bai YY. 2014. Analysis of the results of physical examination of 1638 petroleum workers. Chinese and Foreign Medical Research 12(31): 93-94 
325

326

327

328

329

330

331

332

333

334

335

336

337

338

339

340

341

342

343

344

345

346

347

348

349

350

351

352

353

354

355

356

DOI 10.14033/j.cnki.cfmr.2014.31.124.

Buysse DJ, Rednolds RC, Monk TH, Berman SR, Kupfer DJ. 1989. The Pittsburgh sleep quality index: A new instrument for psychiatric practice and research. Psychiatry Research 28(2): 193-213 DOI 10.1016/0165-1781(89)90047-4.

Tsai PS, Wang SY, Wang MY, Su CT, Yang TT, Huang CJ, Fang SC. 2005. Psychometric evaluation of the Chinese version of the Pittsburgh Sleep Quality Index (CPSQI) in primary insomnia and control subjects. Quality of Life Research. 14(8): 1943-1952

DOI 10.1007/s11136-005-4346-X.

2019. 2018 Chinese guidelines for the management of hypertension. Chinese journal of cardiovascular medicine 24(01): 24-56 DOI 10. 3969/j.issn.1007-5410.2019.01.002.

Huang YL, Mai WY, Cai XY, Hu YZ, Song YB, Qiu RF, Wu YX, Kuang J. 2012. The effect of zolpidem on sleep quality, stress status, and nondipping hypertension. Sleep medicine 13(3): 263-268 DOI 10.1016/j.sleep.2011.07.016.

Lu JP, Lu Y, Wang XC, Li XY, Linderman GC, Wu CQ, Cheng XY, Mu L, Zhang HB, Liu JM, Su M, Zhao HY, Spatz ES, Spertus JA, Masoudi FA, Krumholz HM, Jiang LX. 2017. Prevalence, awareness, treatment, and control of hypertension in China: data from 1.7 million adults in a population-based screening study (China PEACE Million Persons Project). The Lancet 390(10112): 2549-2558 DOI 10.1016/S0140-6736(17)32478-9.

Magnavita N, Di Stasio E, Capitanelli I, Lops EA, Chirico F, Garbarino S. 2019. Sleep Problems and Workplace Violence: A Systematic Review and Meta-Analysis. Front Neuroscience 13:997 DOI 10.3389/fnins.2019.00997.

Tao N, Zhang JJ, Song Z, Tang JH, Liu JW. 2015. Relationship between job burnout and neuroendocrine indicators in soldiers in the Xinjiang arid desert: A cross-sectional study. Multidisciplinary Digital Publishing Institute 12(12): 15154-15161 DOI 10.3390/ijerph121214977.

Zhang HQ, Li YQ, Zhao XY, Mao ZX, Abdulai T, Liu XT, Tu RQ, Wang Y, Qian XI, Jiang JJ, Tian ZY, Luo ZC, Dong XK, Wang CJ, Bie RH. 2019. The association between PSQI score and hypertension in a Chinese rural population: The Henan rural cohort study. Sleep Medicine 58: 27-34 DOI 10.1016/j.sleep.2019.03.001.

Bruno RM, Palagini L, Gemignani A, Virdis A, Giulio AD. 2013. Poor sleep quality and resistant hypertension. Sleep Medicine 14(11):1157-1163 DOI 10.1016/j.sleep.2013.04.020.

Feng X, Liu Q, Li Y, Zhao FF, Chang H, Lyu J. 2019. Longitudinal study of the relationship between sleep duration and hypertension in Chinese adult residents (CHNS 2004-2011). Sleep Medicine 58: 88-92 DOI 10.1016/j.sleep.2019.01.006.

Mong JA, Cusmano DM. 2016. Sex differences in sleep: impact of biological sex and sex steroids. Philosophical transactions of the Royal Society of London. Series B, Biological sciences 371(1688): 20150110 DOI 10.1098/rstb.2015.0110.

Nishichi R, Nufuji Y, Washio M, Kumagai S. 2013. Serum brain-derived neurotrophic factor levels are associated with dyssomnia in females, but not males, among Japanese workers. Journal of clinical sleep medicine 9(7): 649-654 DOI 10.5664/jcsm.2828. 
357

358

359

360

361

362

363

364

365

366

367

368

369

370

371

372

373

374

375

376

377

378

379

380

381

382

383

384

385

386

387

388

389

Hayase M, Shimada M, Seki H. 2014. Sleep quality and stress in women with pregnancy-induced hypertension and gestational diabetes mellitus. Women and Birth 27(3): 190-195 DOI 10.1016/j.wombi.2014.04.002.

Kecklund G, Axelsson J. 2016. Health consequences of shift work and insufficient sleep. BMJ (Clinical research ed.) 355: i5210 DOI 10.1136/bmj.i5210.

Lu K, Jia C, Shouling W, Chen J, Hu DY. 2015. Interaction of sleep duration and sleep quality on hypertension prevalence in adult Chinese males. Journal of epidemiology 25(6): 415-422 DOI 10.2188/jea.JE20140139.

Manohar S, Thongprayoon C, Cheungpasitporn W, Maoa MA, Herrmanna SM Michael A. 2017. Associations of rotational shift work and night shift status with hypertension: A systematic review and meta-analysis. Journal of hypertension 35(10): 1929-1937 DOI 10.1097/HJH.0000000000001442.

Guo Y, Liu Y, Huang X, Rong Y, He MA, Wang YJ,Yuan J, Wu TC, Chen WH. 2017. The effects of shift work on sleeping quality, hypertension and diabetes in retired workers. PLOS ONE 8(8): e71107 DOI 10.1371/journal.pone.0071107.

Härmä M, Karhula K, Ropponen A, Puttonen S, Koskinen A, Ojajärvi A, Hakola T, Pentti J, Oksanen TL, Vahtera, J, Kivimäki M. 2018. Association of changes in work shifts and shift intensity with change in fatigue and disturbed sleep: A within-subject study. Scandinavian journal of work, environment \& health 44(4): 394-402 DOI 10.5271/sjweh.3730.

Heath G, Coates A, Sargent C, Dorrian J. 2016. Sleep duration and chronic fatigue are differently associated with the dietary profile of shift workers. Nutrients $8(12)$ DOI 10.3390/nu8120771.

Elogue EE, Scott ED, Palmieri PA, Dudley P. 2014. Sleep duration, quality, or stability and obesity in an urban family medicine center. Journal of clinical sleep medicine: JCSM: official publication of the American Academy of Sleep Medicine 10(2): 593-598 DOI $10.5664 / \mathrm{jcsm} .3448$.

Magnavita N, Di Stasio E, Capitanelli I, Lops EA, Chirico F, Garbarino S. 2019. Sleep Problems and Workplace Violence: A Systematic Review and Meta-Analysis. Frontiers in Neuroscience 13: 997 DOI 10.3389/fnins.2019.00997.

Garbarino S, Magnavita N. 2019. Sleep problems are a strong predictor of stress-related metabolic changes in police officers. A prospective study. PLOS One 14(10): e0224259 DOI 10.1371/journal.pone.0224259. 


\section{Table $\mathbf{1}$ (on next page)}

The basic characteristics of subjects with or without hypertension 
1 Table 1. The basic characteristics of subjects with or without hypertension $(N=3040)$

\begin{tabular}{|c|c|c|c|c|c|}
\hline \multirow[t]{2}{*}{ Item } & \multirow[t]{2}{*}{ Frequency } & \multicolumn{2}{|c|}{ Hypertension } & \multirow[t]{2}{*}{$\chi^{2}$} & \multirow[t]{2}{*}{ Pvalue } \\
\hline & & Yes & No & & \\
\hline \multicolumn{6}{|l|}{ Sleep duration, $\mathrm{h}$} \\
\hline $7-8$ & 2147 & $283(13.2)$ & $1864(86.8)$ & 26.76 & $<0.001$ \\
\hline$<7$ & 806 & $168(21.0)$ & $638(79.0)$ & & \\
\hline$>8$ & 87 & $15(15.3)$ & $72(84.7)$ & & \\
\hline \multicolumn{6}{|l|}{ Sleep quality } \\
\hline Poor & 2380 & $392(16.5)$ & $1988(83.5)$ & 11.01 & 0.001 \\
\hline Good & 660 & $74(11.2)$ & $586(88.8)$ & & \\
\hline \multicolumn{6}{|l|}{ Gender } \\
\hline Male & 1325 & $134(10.1)$ & $1191 （ 89.9)$ & 49.23 & $<0.001$ \\
\hline Female & 1715 & $332(19.4)$ & $1383(80.6)$ & & \\
\hline \multicolumn{6}{|l|}{ Age, years } \\
\hline$\leq 30$ & 452 & $14(3.4)$ & $438(96.6)$ & 119.79 & $<0.001$ \\
\hline $30-45$ & 1625 & $216(12.9)$ & $1409(87.1)$ & & \\
\hline$>45$ & 963 & $236(24.5)$ & $727(75.5)$ & & \\
\hline \multicolumn{6}{|l|}{ Ethnicity } \\
\hline Han & 2083 & $350(16.8)$ & $1733(83.2)$ & 11.07 & 0.001 \\
\hline Others & 957 & $116(12.1)$ & 841（87.9） & & \\
\hline \multicolumn{6}{|l|}{ Education level } \\
\hline Above senior high school & 1066 & $231(21.7)$ & $835(78.3)$ & 50.86 & $<0.001$ \\
\hline Below technical school & 1974 & $235(11.9)$ & $1739(88.1)$ & & \\
\hline \multicolumn{6}{|l|}{ Job status } \\
\hline Junior level & 865 & $110(12.7)$ & $755(87.3)$ & 34.27 & $<0.001$ \\
\hline Intermediate level & 679 & $70(10.3)$ & $609(89.7)$ & & \\
\hline Senior level & 1496 & $286(9.4)$ & $1210(80.9)$ & & \\
\hline \multicolumn{6}{|l|}{ Marital status } \\
\hline Unmarried & 268 & $20(7.5)$ & $248(92.5)$ & 15.27 & $<0.001$ \\
\hline Married & 2483 & $393(15.8)$ & $2090(84.2)$ & & \\
\hline Divorced/Widow/Widower & 289 & $53(18.3)$ & $236(81.7)$ & & \\
\hline \multicolumn{6}{|l|}{ Income, RMB } \\
\hline$<5000$ & 1580 & $223(14.1)$ & $1357(85.9)$ & 3.74 & 0.053 \\
\hline$\geq 5000$ & 1460 & $243(16.6)$ & $1217(83.4)$ & & \\
\hline \multicolumn{6}{|l|}{ Smoking status } \\
\hline Smoker & 1845 & $243(13.2)$ & $1602(86.8)$ & 16.84 & $<0.001$ \\
\hline Non-smoker & 1195 & $223(18.7)$ & $972(81.3)$ & & \\
\hline \multicolumn{6}{|l|}{ Alcohol consumption } \\
\hline Drinker & 1785 & $319(17.9)$ & $1466(82.1)$ & 21.53 & $<0.001$ \\
\hline Non-drinker & 1255 & $147(11.7)$ & $1108(88.3)$ & & \\
\hline \multicolumn{6}{|l|}{ Shift work status } \\
\hline Shift work & 1503 & $283(18.8)$ & $1220(81.2)$ & 28.06 & $<0.001$ \\
\hline No shift work & 1537 & $183(11.9)$ & $1354(88.1)$ & & \\
\hline \multicolumn{6}{|c|}{ BMI, $\mathrm{kg} \cdot \mathrm{m}^{-2}\left(\right.$ Body mass index, $\left.\mathrm{kg} \cdot \mathrm{m}^{-2}\right)$} \\
\hline$<18.5$ & 84 & $3(3.6)$ & $81(96.4)$ & 104.45 & $<0.001$ \\
\hline $18.5-24$ & 1604 & $156(9.7)$ & $1448(90.3)$ & & \\
\hline$>24$ & 1352 & $307(22.7)$ & $1045(77.3)$ & & \\
\hline Length of service, years & & & & & \\
\hline
\end{tabular}




\begin{tabular}{cccccc}
\hline$<10$ & 850 & $35(4.1)$ & $815(95.9)$ & 141.32 & $<0.001$ \\
$10-20$ & 566 & $73(12.9)$ & $493(87.1)$ & & \\
$>20$ & 1624 & $358(22.0)$ & $1266(78.0)$ & & \\
\hline
\end{tabular}

2 


\section{Table 2 (on next page)}

Results of logistic regression analysis investigating the association between sleep duration, sleep quality and the risk of hypertension among petroleum workers 
1 Table 2 Results of logistic regression analysis investigating the association between sleep duration, sleep quality and the risk of hypertension among oil workers

\begin{tabular}{|c|c|c|c|c|c|}
\hline Item & $b$ & $S E$ & Wald $\chi^{2}$ & $P$ value & OR $(95 \% C I)$ \\
\hline \multicolumn{6}{|l|}{ Sleep duration, $\mathrm{h}$} \\
\hline $7-8$ & - & - & - & - & 1.00 \\
\hline$<7$ & 0.409 & 0.119 & 11.785 & 0.001 & $1.51(1.19-1.90)$ \\
\hline$>8$ & 0.424 & 0.318 & 1.772 & 0.183 & $1.53(0.82-2.85)$ \\
\hline \multicolumn{6}{|l|}{ Sleep quality } \\
\hline Good & - & - & - & - & 1.00 \\
\hline Poor & 0.576 & 0.148 & 15.166 & $<0.001$ & $1.78(1.33-2.38)$ \\
\hline \multicolumn{6}{|l|}{ Gender } \\
\hline Male & - & - & - & - & 1.00 \\
\hline Female & 0.730 & 0.154 & 22.389 & $<0.001$ & $2.08(1.53-2.81)$ \\
\hline \multicolumn{6}{|l|}{ Age, years } \\
\hline$<30$ & - & - & - & - & 1.00 \\
\hline $30-45$ & 0.694 & 0.342 & 4.125 & 0.042 & $2.00(1.03-3.91)$ \\
\hline$>45$ & 1.066 & 0.362 & 8.675 & 0.003 & $2.91(1.43-5.91)$ \\
\hline \multicolumn{6}{|l|}{ Ethnicity } \\
\hline Han & - & - & - & - & 1.00 \\
\hline Others & -0.498 & 0.132 & 14.227 & $<0.001$ & $0.61(0.50-0.79)$ \\
\hline \multicolumn{6}{|l|}{ Educational level } \\
\hline Above senior high school & - & - & - & - & 1.00 \\
\hline Below technical school & -0.199 & 0.121 & 2.684 & 0.101 & $0.82(0.65-1.04)$ \\
\hline \multicolumn{6}{|l|}{ Job status } \\
\hline Junior level & - & - & - & - & 1.00 \\
\hline Intermediate level & -0.300 & 0.158 & 3.602 & 0.058 & $0.74(0.54-1.01)$ \\
\hline Senior level & -0.050 & 0.135 & 0.138 & 0.710 & $0.95(0.73-1.24)$ \\
\hline \multicolumn{6}{|l|}{ Marital status } \\
\hline Unmarried & - & - & - & - & 1.00 \\
\hline Married & -0.305 & 0.293 & 1.086 & 0.297 & $0.74(0.42-1.31)$ \\
\hline Divorced/Widow/Widower & -0.095 & 0.333 & 0.082 & 0.755 & $0.91(0.47-1.75)$ \\
\hline \multicolumn{6}{|l|}{ Income, RMB } \\
\hline$<5000$ & - & - & - & - & 1.00 \\
\hline$\geq 5000$ & -0.427 & 0.112 & 14.580 & $<0.001$ & $0.65(0.52-0.81)$ \\
\hline \multicolumn{6}{|l|}{ Smoking status } \\
\hline Non-smoker & - & - & - & - & 1.00 \\
\hline Smoker & -1.360 & 0.136 & 1.011 & 0.315 & $0.87(0.70-1.14)$ \\
\hline \multicolumn{6}{|l|}{ Alcohol consumption } \\
\hline Non-drinker & - & - & - & - & 1.00 \\
\hline Drinker & 0.173 & 0.131 & 1.742 & 0.187 & $1.19(0.92-1.54)$ \\
\hline \multicolumn{6}{|l|}{ Shift work status } \\
\hline No shift work & - & - & - & - & 1.00 \\
\hline Shift work & 0.797 & 0.114 & 48.849 & $<0.001$ & $2.22(1.78-2.78)$ \\
\hline \multicolumn{6}{|c|}{ BMI, $\mathrm{kg} \cdot \mathrm{m}^{-2}\left(\right.$ Body mass index, $\left.\mathrm{kg} \cdot \mathrm{m}^{-2}\right)$} \\
\hline $18.5 \sim 24$ & - & — & - & - & 1.00 \\
\hline$<18.5$ & -0.018 & 0.620 & 0.001 & 0.977 & $0.98(0.29-3.31)$ \\
\hline$>24$ & 0.883 & 0.118 & 55.753 & $<0.001$ & $2.42(1.92-3.05)$ \\
\hline \multicolumn{6}{|l|}{ Length of service, years } \\
\hline$<10$ & - & - & - & - & 1.00 \\
\hline $10-20$ & 0.849 & 0.250 & 11.541 & 0.001 & $2.34(1.43-3.81)$ \\
\hline$>20$ & 1.354 & 0.243 & 30.941 & $<0.001$ & $3.87(2.40-6.24)$ \\
\hline
\end{tabular}




\section{Table 3(on next page)}

Relationship between sleep duration, sleep quality and hypertension after gender stratification 
1 Table 3 Relationship between sleep duration, sleep quality and hypertension after stratification

\begin{tabular}{|c|c|c|c|c|c|c|c|c|}
\hline \multirow[t]{2}{*}{ Item } & \multicolumn{5}{|c|}{ Sleep duration, $\mathrm{h}$} & \multicolumn{3}{|c|}{ Sleep quality } \\
\hline & $7-8$ & $<7$ & $P$ & $>8$ & $P$ & Good & Poor & $P$ \\
\hline \multicolumn{9}{|l|}{ Gender $^{\mathrm{a}}$} \\
\hline Female & 1.00 & $1.54(1.16-2.04)$ & 0.003 & $1.62(0.75-3.47)$ & 0.218 & 1.00 & $1.78(1.26-2.49)$ & 0.001 \\
\hline Male & 1.00 & $1.49(1.00-2.23)$ & 0.049 & $1.63(0.58-4.58)$ & 0.350 & 1.00 & $1.47(0.88-2.46)$ & 0.142 \\
\hline \multicolumn{9}{|l|}{ Age, years b } \\
\hline$<30$ & 1.00 & $9.03(2.32-35.15)$ & 0.002 & $3.89(0.27-42.08)$ & 0.342 & 1.00 & $5.24(0.35-78.90)$ & 0.232 \\
\hline $30-45$ & 1.00 & $1.59(1.14-2.20)$ & 0.006 & $0.79(0.23-2.68)$ & 0.707 & 1.00 & $1.71(1.10-2.66)$ & 0.017 \\
\hline$>45$ & 1.00 & $1.27(0.91-1.77)$ & 0.161 & $3.36(1.42-7.91)$ & 0.006 & 1.00 & $1.60(1.09-2.34)$ & 0.016 \\
\hline \multicolumn{9}{|c|}{ Shift work status ${ }^{c}$} \\
\hline Shift work & 1.00 & $1.55(1.16-2.07)$ & 0.003 & $0.98(0.39-2.46)$ & 0.967 & 1.00 & $1.70(1.17-2.47)$ & 0.005 \\
\hline No shift work & 1.00 & $1.48(1.02-2.15)$ & 0.039 & $1.92(0.76-4.82)$ & 0.165 & 1.00 & $1.60(1.03-2.48)$ & 0.037 \\
\hline \multicolumn{9}{|c|}{ Length of service, years ${ }^{d}$} \\
\hline$<10$ & 1.00 & $4.08(1.92-8.83)$ & $<0.001$ & $1.68(0.35-8.11)$ & 0.520 & 1.00 & $0.74(0.31-1.74)$ & 0.488 \\
\hline $10-20$ & 1.00 & $2.79(1.59-4.86)$ & $<0.001$ & $0.82(0.10-6.83)$ & 0.855 & 1.00 & $3.01(1.23-7.35)$ & 0.016 \\
\hline$>20$ & 1.00 & $1.20(0.92-1.56)$ & 0.191 & $2.25(1.06-4.79)$ & 0.035 & 1.00 & $1.64(1.18-2.27)$ & 0.003 \\
\hline
\end{tabular}

a Adjust factors: age, ethnicity, income and shift work status, length of service

b Adjust factors: gender, ethnicity, income and shift work status, length of service

${ }^{c}$ Adjust factors: age, gender, ethnicity, income and length of service

${ }^{\mathrm{d}}$ Adjust factors: age, gender, ethnicity, income and shift work status

The $O R$ value and $95 \%$ confidence interval are listed in the table. 